\title{
Irregular varieties of Albanese fiber dimension one with small volume
}

TONG ZHANG

In this paper, we generalize Horikawa's theorem on irregular surfaces with small $c_{1}^{2}$ to higher dimensional irregular varieties of Albanese fiber dimension one with small volume.

\section{Introduction}

This paper is motivated by the following theorem of Horikawa:

Theorem. [7, Theorem 3.1] Let $S$ be an irregular minimal surface of general type over $\mathbb{C}$.

(1) If $\frac{8}{3} \chi\left(\mathcal{O}_{S}\right) \leq K_{S}^{2}<3 \chi\left(\mathcal{O}_{S}\right)$, then the Albanese map $S \rightarrow \operatorname{Alb}(S)$ induces a hyperelliptic pencil of curves of genus 2 or 3 .

(2) If $2 \chi\left(\mathcal{O}_{S}\right) \leq K_{S}^{2}<\frac{8}{3} \chi\left(\mathcal{O}_{S}\right)$, then the Albanese map $S \rightarrow \operatorname{Alb}(S)$ induces a pencil of curves of genus 2 .

The above theorem characterizes the geometry of irregular surfaces with small volume. It plays a crucial role in the theory of algebraic surfaces. For example, it leads to the following important conjecture proposed by Reid in [10]:

Conjecture (Reid). For $g=2,3, \ldots$, there exist rational numbers $a_{g}$ and $b_{g}$ with

$$
a_{2}<a_{3}<\cdots \quad \text { and } \quad \lim _{g \rightarrow \infty} a_{g}=4
$$

such that for every surface $X$ of general type, if $K_{X}^{2} \leq a_{g} \chi\left(\mathcal{O}_{X}\right)-b_{g}$, then $X$ has a pencil of curves of genus at most $g$.

For this conjecture, to our knowledge, only $a_{2}=\frac{8}{3}$ and $a_{3}=3$ are known. Partial results are also known. We refer to $[10,12]$ for details about this conjecture. 
In fact, it is well-known that the relation between the (canonical) volume and the holomorphic Euler characteristic $\chi\left(\mathcal{O}_{X}\right)$ is a fundamental problem in the study of algebraic varieties, in particular, in the geography of algebraic varieties. Our purpose in this paper is to generalize Horikawa's theorem to higher dimensions. Throughout this paper, we will work over an algebraically closed field of characteristic zero.

Our main theorem is the following:

Theorem 1.1. Let $X$ be an $n$-dimensional normal, irregular minimal $\mathbb{Q}$ Gorenstein variety of general type with Albanese fiber dimension one.

(1) If $\frac{8}{3}(n-1) ! \chi\left(\omega_{X}\right) \leq K_{X}^{n}<3(n-1) ! \chi\left(\omega_{X}\right)$, then the Albanese map $X \rightarrow \operatorname{Alb}(X)$ induces a fibration of hyperelliptic curves of genus 2 or 3 .

(2) If $2(n-1) ! \chi\left(\omega_{X}\right) \leq K_{X}^{n}<\frac{8}{3}(n-1) ! \chi\left(\omega_{X}\right)$, then the Albanese map $X \rightarrow \operatorname{Alb}(X)$ induces a fibration of curves of genus 2 .

When $n=2$, the above theorem coincides with Horikawa's theorem.

Notice that for an irregular minimal surface $S$ of general type, we always have $K_{S}^{2} \geq 2 \chi\left(\mathcal{O}_{S}\right)$ by Bombieri [4]. ${ }^{1}$ This result is recently generalized by Barja in [1, Remark 4.5], where he has shown that $K_{X}^{n} \geq 2(n-1) ! \chi\left(\omega_{X}\right)$ for any Gorenstein variety $X$ satisfying the hypothesis in Theorem 1.1. In fact, using the method in this paper, we can give another proof of Barja's result. See Section 6.

It is also worth pointing out that if $X$ is of maximal Albanese dimension, it is recently proved by Barja [1] and independently by the author [14] that we have the generalized Severi inequality $K_{X}^{n} \geq 2 n ! \chi\left(\omega_{X}\right)$. When $n=2$, it coincides with the original Severi inequality proved by Pardini [9].

Before outlining the proof of Theorem 1.1, we first recall the original proof of Horikawa's theorem. Horikawa's original proof is as follows: using the Castelnuovo inequality $K^{2} \geq 3 p_{g}-7$, the problem can be reduced to hyperelliptic fibrations. Then by the double cover method, the ratio $K^{2} / \chi\left(\mathcal{O}_{X}\right)$ can be explicitly calculated with a lower bound $4-\frac{4}{g}$, where $g$ is the genus of Albanese fibers. A different treatment is to apply Xiao's slope inequality in [11], which also gives the same lower bound. Unfortunately, in the higher dimensional case, the double cover method seems to be hard to handle. Also, to the best of our knowledge, the slope inequality for higher dimensional families of curves is still unknown.

Our proof for arbitrary dimension is different from the above. We observe that, with the help of Pardini's covering method [9] and the generic

\footnotetext{
${ }^{1}$ In fact, we even have a stronger result $K_{S}^{2} \geq 2 p_{g}(S)$ due to Debarre [5].
} 
vanishing theorem of Green and Lazarsfeld [3], we can reduce this question to the explicit comparison between $h^{0}\left(K_{X}\right)$ and $K_{X}^{n}$ on $X$ with a fibration of relative dimension one, which is Theorem 4.6. The first two inequalities in Theorem 4.6 concern the hyperelliptic case. While, the last one plays the role of the Castelnuovo inequality in the surface case, which helps exclude the non-hyperelliptic case.

To prove Theorem 4.6, we study a more general comparison between $h^{0}(L)$ and $\left(\sigma^{*} K_{X}\right) L^{n-1}$ for any nef Cartier divisor $L \leq \sigma^{*} K_{X}$ on $X^{\prime}$. Here $\sigma: X^{\prime} \rightarrow X$ is any birational morphism. First, we use Proposition 2.4 and numerical results in [13] to get the desired ratios between $h^{0}(L)$ and $\left(\sigma^{*} K_{X}\right) L$ under different genus restrictions on fibered surfaces, i.e., when $n=2$ (see Theorem 3.2). Finally, by induction on the dimension, we can finish the proof.

\section{Conventions}

Throughout this paper, $X$ is always a projective variety. We say that $X$ is minimal $\mathbb{Q}$-Gorenstein of general type, if $X$ has at worst terminal singularities and $K_{X}$ is a nef and big $\mathbb{Q}$-Cartier divisor. We say $X$ is irregular, if $h^{1}\left(\mathcal{O}_{X}\right)>0$, i.e., $X$ has nontrivial Albanese map. For $X$ irregular, we say that $X$ has one dimensional Albanese fiber, if its Albanese image has dimension $\operatorname{dim} X-1$.

Let $X$ be a projective variety of dimension $n \geq 2$. We say that there is a family of hyperelliptic (resp. non-hyperelliptic, $\mathbb{P}^{1}$ ) curves on $X$, if there exists a variety $Y$ of dimension $n-1$ and a rational map $f: X \rightarrow Y$ such that the general fibers of $f$ are hyperelliptic (resp. non-hyperelliptic, $\mathbb{P}^{1}$ ) curves. When $\operatorname{dim} Y=1$, such a family is also called a pencil. It is called a fibration, if $f$ is a morphism.

\section{Preliminaries}

Let $X$ be a smooth projective variety of dimension $n \geq 2$. Let $f: X \rightarrow C$ be a fibration from $X$ to a smooth projective curve $C$ with a smooth general fiber $F$. For any nef Cartier divisor $L$ on $X$, we can find a unique integer $e_{L}$ such that

- $L-e_{L} F$ is not nef;

- $L-e F$ is nef for any integer $e<e_{L}$. 
We call this number the minimum of $L$ with respect to $F$. In particular, $e_{L}>0$.

Theorem 2.1. With the above notation. Let $L$ be a nef and effective Cartier divisor on $X$. Then we have the following quadruples

$$
\left\{\left(X_{i}, L_{i}, Z_{i}, a_{i}\right), \quad i=0,1, \ldots, N\right\}
$$

with the following properties:

1) $\left(X_{0}, L_{0}, Z_{0}, a_{0}\right)=\left(X, L, 0, e_{L}\right)$.

2) For any $i=0, \ldots, N-1, \pi_{i}: X_{i+1} \rightarrow X_{i}$ is a composition of blowups of $X_{i}$ such that the proper transform of the movable part of $\mid L_{i}-$ $a_{i} F_{i} \mid$ is base point free. Here $F_{0}=F, F_{i+1}=\pi_{i}^{*} F_{i}$, and $a_{i}=e_{L_{i}}$ is the minimum of $L_{i}$ with respect to $F_{i}$. Moreover, we have the decomposition

$$
\pi_{i}^{*}\left(L_{i}-a_{i} F_{i}\right)=L_{i+1}+Z_{i+1}
$$

such that $\left|L_{i+1}\right|$ is base point free and $Z_{i+1} \geq 0$.

3) We have $h^{0}\left(L_{0}\right) \geq h^{0}\left(L_{1}\right)>\cdots>h^{0}\left(L_{N}\right)>h^{0}\left(L_{N}-a_{N} F_{N}\right)=0$. Here $a_{N}=e_{L_{N}}$.

Proof. See [14, Theorem 2.3].

Remark 2.2. If $\operatorname{dim} X=2$, by [13, Theorem 2.2], we can assume $X_{i}=X_{0}$ for any $i$, i.e., we do not need blow-ups. In this case, $\left|L_{i+1}\right|$ may not be base point free, but it has no fixed part (see [13, Theorem 2.2 (2)]). Hence its base locus has dimension 0 (or empty). Still by [13, Theorem 2.2 (4)], we know that

$$
L_{0} F>L_{1} F>\cdots>L_{N} F \geq 0 .
$$

If $\left|L_{0}\right|$ itself has only isolated base points, then $\left|L_{i}\right|_{F} \mid$ is base point free. Thus

$$
h^{0}\left(\left.L_{0}\right|_{F}\right)>h^{0}\left(\left.L_{1}\right|_{F}\right)>\cdots>h^{0}\left(\left.L_{N}\right|_{F}\right) \geq 1 .
$$

In particular, it implies that

$$
N \leq h^{0}\left(\left.L_{0}\right|_{F}\right)-1 .
$$

This will be applied in the proof of Theorem 3.2 in Section 3 . 
Write $\rho_{i}=\pi_{0} \circ \cdots \circ \pi_{i-1}: X_{i} \rightarrow X_{0}$ for $i=1, \ldots, N$ and let $\rho_{0}$ be the identity morphism from $X_{0}$ to itself. Denote

$$
L_{i}^{\prime}=L_{i}-a_{i} F_{i}, \quad r_{i}=h^{0}\left(\left.L_{i}\right|_{F_{i}}\right)
$$

Fix a nef $\mathbb{Q}$-Cartier divisor $P$ on $X$ and write $P_{i}=\rho_{i}^{*} P$. Denote

$$
d_{i}= \begin{cases}\left(\left.P_{i}\right|_{F_{i}}\right)\left(\left.L_{i}\right|_{F_{i}}\right)^{n-2}, & n \geq 3 \\ P_{i} F_{i}, & n=2\end{cases}
$$

Remark 2.3. It is worth noticing that if $n=2$, then $d_{0}=d_{1}=d_{2}=\cdots$. The reader can be also reminded of this observation during the proof of Theorem 3.2 in Section 3.

Proposition 2.4. For any $j=0,1, \ldots, N$, we have the following numerical inequalities:

(1) $h^{0}\left(L_{0}\right) \leq h^{0}\left(L_{j}^{\prime}\right)+\sum_{i=0}^{j} a_{i} r_{i}$

(2) $P_{0} L_{0}^{n-1} \geq(n-1) \sum_{i=0}^{j} a_{i} d_{i}-(n-1) d_{0}$.

Proof. In [14, Proposition 2.6], (1) is proved. For (2), since $P_{i}, L_{i}^{\prime}+F_{i}$, and $L_{i+1}^{\prime}+F_{i+1}$ are all nef, we have

$$
\begin{aligned}
P_{i}\left(L_{i}^{\prime}+F_{i}\right)^{n-1} & =P_{i+1}\left(\pi_{i}^{*} L_{i}^{\prime}+F_{i+1}\right)^{n-1} \\
& \geq P_{i+1}\left[\left(L_{i+1}^{\prime}+F_{i+1}\right)+a_{i+1} F_{i+1}\right]^{n-1} \\
& =P_{i+1}\left(L_{i+1}^{\prime}+F_{i+1}\right)^{n-1}+(n-1) a_{i+1} d_{i+1} .
\end{aligned}
$$

Summing over $i=0, \ldots, j-1$, we have

$$
P_{0}\left(L_{0}^{\prime}+F_{0}\right)^{n-1} \geq(n-1) \sum_{i=1}^{j} a_{i} d_{i}+P_{j}\left(L_{j}^{\prime}+F_{j}\right)^{n-1} \geq(n-1) \sum_{i=1}^{j} a_{i} d_{i}
$$

Note that

$$
P_{0}\left(L_{0}^{\prime}+F_{0}\right)^{n-1}=P_{0} L_{0}^{n-1}-(n-1)\left(a_{0}-1\right) d_{0}
$$

This finishes the proof. 
Lemma 2.5. Under the above setting, we have

$$
P_{0} L_{0}^{n-1} \geq d_{0}\left((n-1)\left(a_{0}-1\right)+\sum_{i=1}^{N} a_{i}\right) \geq d_{0}\left(\sum_{i=0}^{N} a_{i}-1\right) .
$$

Proof. For $i=0, \ldots, N-1$, denote by

$$
\tau_{i}=\pi_{i} \circ \cdots \circ \pi_{N-1}: X_{N} \rightarrow X_{i}
$$

the composition of blow-ups and let $\tau_{N}=\operatorname{id}_{X_{N}}: X_{N} \rightarrow X_{N}$.

Write $b=a_{1}+\cdots+a_{N}$ and $Z=\tau_{1}^{*} Z_{1}+\cdots+\tau_{N}^{*} Z_{N}$. We have the following numerical equivalence on $X_{N}$ :

$$
\tau_{0}^{*} L_{0}^{\prime} \sim_{\text {num }} L_{N}^{\prime}+b F_{N}+Z .
$$

Since $L_{0}^{\prime}+F_{0}$ and $L_{N}^{\prime}+F_{N}$ are both nef, it follows that

$$
\begin{aligned}
P_{0}\left(L_{0}^{\prime}+F_{0}\right)^{n-1} & =P_{N}\left(\tau_{0}^{*} L_{0}^{\prime}+F_{N}\right)^{n-2}\left(L_{N}^{\prime}+F_{N}+b F_{N}+Z\right) \\
& \geq b P_{N}\left(\tau_{0}^{*} L_{0}^{\prime}+F_{N}\right)^{n-2} F_{N} \\
& =b d_{0} .
\end{aligned}
$$

Note that

$$
P_{0} L_{0}^{n-1}-P_{0}\left(L_{0}^{\prime}+F_{0}\right)^{n-1}=(n-1)\left(a_{0}-1\right) d_{0} .
$$

Hence the first inequality is proved. Since $a_{0} \geq 1$, the second inequality holds.

\section{Linear series on algebraic surfaces}

In this section, we set up some basic results about linear series on algebraic surfaces. Throughout this section, we will use the following assumptions:

(A3.1) $S^{\prime}$ is a minimal surface of general type, and $\sigma: S \rightarrow S^{\prime}$ is a birational morphism.

(A3.2) $L$ is a nef Cartier divisor on $S$, and $P$ is a nef $\mathbb{Q}$-Cartier divisor on $S$ such that $L \leq P \leq \sigma^{*} K_{S^{\prime}}$.

Proposition 3.1. Assume that $S$ has no hyperelliptic pencil. Then

$$
\left(\sigma^{*} K_{S^{\prime}}\right) L \geq 3 h^{0}(L)-7 .
$$


Proof. First, we can assume that $h^{0}(L) \geq 3$. Otherwise, the result would become trivial. Replacing $S$ by an appropriate resolution, we can further assume that $|L|$ is base point free. Denote by $\phi_{L}: S \rightarrow \mathbb{P}^{h^{0}}(L)-1$ the morphism induced by $|L|$.

If $\phi_{L}$ is generically finite and $L^{2}<3 h^{0}(L)-7$, then using exactly the same argument in [2, Théorème 5.5], we know that $S$ is a double cover over a birationally ruled surface. This would imply that $S$ has a hyperelliptic pencil, which is impossible. Hence in this case, we have

$$
\left(\sigma^{*} K_{S^{\prime}}\right) L \geq L^{2} \geq 3 h^{0}(L)-7
$$

When $\operatorname{dim} \phi_{L}(S)=1$, we can write

$$
L \sim_{\text {num }} r C
$$

where $C$ is a general fiber of $\phi_{L}$, and $r \geq h^{0}(L)-1 \geq 2$. If $\left(\sigma^{*} K_{S^{\prime}}\right) C \geq 3$, then

$$
\left(\sigma^{*} K_{S^{\prime}}\right) L \geq 3 r>3 h^{0}(L)-7 .
$$

If $\left(\sigma^{*} K_{S^{\prime}}\right) C \leq 2$, then $K_{S^{\prime}} C^{\prime} \leq 2$, where $C^{\prime}=\sigma(C)$. On the other hand, since $L \leq \sigma^{*} K_{S^{\prime}}$, we know that $K_{S^{\prime}}-2 C^{\prime}$ is pseudo-effective. Hence

$$
C^{\prime 2} \leq \frac{K_{S^{\prime}} C^{\prime}}{2} \leq 1
$$

By parity, the only possibility is that $K_{S^{\prime}} C^{\prime}+C^{\prime 2}=2$, i.e., $p_{a}\left(C^{\prime}\right)=2$. But it is absurd, because $C^{\prime}$ can not be hyperelliptic.

Theorem 3.2. Assume that $S$ has a hyperelliptic fibration of genus $g$ curves and $P C=2 g-2$, where $C$ is a general fiber.

(1) If $g \geq 3$, then

$$
P L \geq \frac{8}{3} h^{0}(L)-(4 g-4)
$$

(2) If $g \geq 4$, then

$$
P L \geq 3 h^{0}(L)-(4 g-4)
$$

Proof. Replacing $S$ by an appropriate resolution, we can assume that $|L|$ is base point free. Since $L \leq \sigma^{*} K_{S^{\prime}} \leq K_{S}$, by adjunction, $\left.L\right|_{C} \leq K_{C}$, i.e., $\left.L\right|_{C}$ is a special divisor. Moreover, since $C$ is hyperelliptic and $|L|_{C} \mid$ is base point free, by [13, Lemma 2.6], we know that $L C$ has to be even. 
Let us prove (1) first. Assume that $g \geq 3$. If $L C \geq 4$, then we have

$$
P L \geq L^{2} \geq \frac{4 L C}{L C+2} h^{0}(L)-2 L C \geq \frac{8}{3} h^{0}(L)-(4 g-4) .
$$

The second inequality is by [13, Theorem 1.1], and the third inequality follows from the fact that $L C \leq P C=2 g-2$. If $L C=0$, we know that $L \sim_{\text {num }} r C$, where $r \geq h^{0}(L)-1$. Thus

$$
P L=r P C \geq(2 g-2)\left(h^{0}(L)-1\right) \geq 4 h^{0}(L)-4>4 h^{0}(L)-(4 g-4) .
$$

The only issue here is when $L C=2$. In this case, since $r_{0}=h^{0}\left(\left.L\right|_{C}\right)=2$, we can apply Theorem 2.1 and Remark 2.2 to $L$ to get the following triples

$$
\left\{\left(L_{i}, Z_{i}, a_{i}\right): i=0, \ldots, N\right\}
$$

where $N \leq 1$. Assume first that we get two triples, i.e., $N=1$. By Remark 2.2, we know that $r_{1}=h^{0}\left(\left.L_{1}\right|_{C}\right)=1$ and that $\left|L_{1}\right|_{C} \mid$ is base point free. It forces $\left.L_{1}\right|_{C}=\mathcal{O}_{C}$. We will divide the proof into two cases.

Case 1.1: $a_{0} \leq a_{1}$. By Remark 2.3 and Proposition 2.4, we get

$$
\begin{aligned}
h^{0}(L) & \leq a_{0} r_{0}+a_{1} r_{1}=2 a_{0}+a_{1}, \\
P L & \geq a_{0} d_{0}+a_{1} d_{1}-d_{0} \geq 4 a_{0}+4 a_{1}-(2 g-2) .
\end{aligned}
$$

It follows that

$$
\frac{P L+(4 g-4)}{h^{0}(L)}>\frac{P L+(2 g-2)}{h^{0}(L)} \geq \frac{4 a_{0}+4 a_{1}}{2 a_{0}+a_{1}} \geq \frac{8}{3} .
$$

Case 1.2: $a_{0}>a_{1}$. In this case, instead of using Proposition 2.4, we need to use [14, Proposition 2.3], by which we obtain

$$
L^{2} \geq 2 a_{0} d_{0}^{\prime}+\left(d_{0}^{\prime}+d_{1}^{\prime}\right) a_{1}-2 d_{0}^{\prime}=4 a_{0}+2 a_{1}-4 .
$$

Here $d_{i}^{\prime}=L_{i} C$ so that $d_{0}^{\prime}=2$ and $d_{1}^{\prime}=0$.

Notice that $L-\left(a_{0}-1\right) C$ is nef. Thus

$$
\begin{aligned}
(P-L) L & =(P-L)\left(L-\left(a_{0}-1\right) C\right)+\left(a_{0}-1\right)(P-L) C \\
& \geq\left(a_{0}-1\right)(2 g-4) \geq 2 a_{0}-2 .
\end{aligned}
$$

Combine this with (3.3), and we get

$$
P L=L^{2}+\left(P L-L^{2}\right) \geq 6 a_{0}+2 a_{1}-6 .
$$


On the other hand, the inequality (3.1) in Case 1.1 still holds in this case. Hence

$$
\frac{P L+(4 g-4)}{h^{0}(L)}>\frac{P L+6}{h^{0}(L)} \geq \frac{6 a_{0}+2 a_{1}}{2 a_{0}+a_{1}}>\frac{8}{3} .
$$

Assume now that we only have one triple, i.e., $N=0$. In this case, the above proof still applies by letting $a_{1}=0$ and the result follows. ${ }^{2}$

Now let us prove (2). In fact, the strategy is quite similar but a bit more complicated. In the following, we will assume that $g \geq 4$.

When $L C \geq 6$, by [13, Theorem 1.1] again, we get

$$
P L \geq L^{2} \geq \frac{4 L C}{L C+2} h^{0}(L)-2 L C \geq 3 h^{0}(L)-(4 g-4) .
$$

When $L C=0$, we can just apply a very similar argument as above to deduce that

$$
P L \geq(2 g-2)\left(h^{0}(L)-1\right) \geq 6 h^{0}(L)-(2 g-2)>3 h^{0}(L)-(4 g-4) .
$$

When $L C=2$, using the "at most two triples" method verbatim as before and by Remark 2.3 and Proposition 2.4, we have

$$
\begin{aligned}
h^{0}(L) & \leq a_{0} r_{0}+a_{1} r_{1}=2 a_{0}+a_{1}, \\
P L & \geq a_{0} d_{0}+a_{1} d_{1}-d_{0} \geq 6 a_{0}+6 a_{1}-(2 g-2) .
\end{aligned}
$$

Hence

$$
P L \geq 3 h^{0}(L)-(2 g-2)>3 h^{0}(L)-(4 g-4) .
$$

It remains to consider the case when $L C=4$. In this case, since $r_{0}=$ $h^{0}\left(\left.L\right|_{C}\right)=3$, by Theorem 2.1 and Remark 2.2, we can get the following triples

$$
\left\{\left(L_{i}, Z_{i}, a_{i}\right): i=0, \ldots, N\right\}
$$

where $N \leq 2$. We first assume that we get three triples, i.e., $N=2$. Then both $\left|L_{1}\right|_{C} \mid$ and $\left|L_{2}\right|_{C} \mid$ are base point free. Note that $r_{1}=h^{0}\left(\left.L_{1}\right|_{C}\right)=2$ and $r_{2}=h^{0}\left(\left.L_{2}\right|_{C}\right)=1$. It is easy to see that $\left.L_{1}\right|_{C}=g_{2}^{1}$ and $\left.L_{2}\right|_{C}=\mathcal{O}_{C}$. We divide the proof into two cases again.

\footnotetext{
${ }^{2}$ In fact, in this case, we only need to use the argument in Case 1.2.
} 
Case 2.1: $a_{0} \leq a_{2}$. In this case, by Remark 2.3 and Proposition 2.4, we have

$$
\begin{aligned}
h^{0}(L) & \leq \sum_{i=0}^{2} a_{i} r_{i}=3 a_{0}+2 a_{1}+a_{2} \\
P L & \geq \sum_{i=0}^{2} a_{i} d_{i}-d_{0} \geq 6\left(a_{0}+a_{1}+a_{2}\right)-(2 g-2) .
\end{aligned}
$$

Hence

$$
P L+(4 g-4)>6\left(a_{0}+a_{1}+a_{2}\right) \geq 3\left(3 a_{0}+2 a_{1}+a_{2}\right) \geq 3 h^{0}(L) .
$$

Case 2.2: $a_{0}>a_{2}$. We follow the idea in Case 1.2 once more. By [15, Proposition 2.3], we have

$$
L^{2} \geq 2 a_{0} d_{0}^{\prime}+\sum_{i=1}^{2}\left(d_{i-1}^{\prime}+d_{i}^{\prime}\right) a_{i}-2 d_{0}^{\prime}=8 a_{0}+6 a_{1}+2 a_{2}-8 .
$$

Here $d_{i}^{\prime}=L_{i} C$ so that $d_{0}^{\prime}=4, d_{1}^{\prime}=2$, and $d_{2}^{\prime}=0$.

By a similar argument as in Case 1.2, we can get

$$
L(P-L) \geq\left(a_{0}-1\right)(P-L) C=\left(a_{0}-1\right)(2 g-6) \geq 2 a_{0}-2 .
$$

Hence

$$
\begin{aligned}
P L & =L^{2}+L(P-L) \geq 10 a_{0}+6 a_{1}+2 a_{2}-10 \\
& >3\left(3 a_{0}+2 a_{1}+a_{2}\right)-10>3 h^{0}(L)-(4 g-4) .
\end{aligned}
$$

If we only get one triple or two, the proof here still applies after suitable modifications. We just list the modifications and leave the proof to the reader:

- If $N=0$, set $a_{1}=a_{2}=d_{1}^{\prime}=d_{2}^{\prime}=0$;

- If $N=1$ and $\left.L_{1}\right|_{C}=g_{2}^{1}$, set $a_{2}=d_{2}^{\prime}=0$;

- If $N=1$ and $\left.L_{1}\right|_{C}=\mathcal{O}_{C}$, set $a_{1}=d_{1}^{\prime}=d_{2}^{\prime}=0$.

\section{Linear series on varieties fibered by curves}

In this section, we will generalize the results in Section 3 to arbitrary dimension. 
Assumption 4.1. Throughout this section, we assume that:

- $X$ is a projective, normal and minimal $\mathbb{Q}$-Gorenstein variety of general type, and $L \leq K_{X}$ is a nef Cartier divisor on $X$.

- $f: X \rightarrow Y$ is a fibration of genus $g \geq 2$ from $X$ to a normal projective variety $Y$ of dimension $n-1$ with connected fibers, and $C$ is a general fiber.

- $M$ is a Cartier divisor on $Y$ such that $M^{n-1}>0$ and $|M|$ is base point free. Write $B=f^{*} M$.

- Since $h^{0}\left(l K_{X}\right)>0$ for $l \in \mathbb{N}$ sufficiently large, we write

$$
K_{X} \sim_{\operatorname{lin}_{\mathbb{Q}}} \sum_{i=1}^{I_{0}} q_{i} H_{i}+V
$$

up to $\mathbb{Q}$-linear equivalence. Here $q_{i} \in \mathbb{Q}_{>0}$, each $H_{i} \geq 0$ is an irreducible, reduced and horizontal Cartier divisor, and $V \geq 0$ is the vertical part. Fix an integer $k>0$ such that $\left.\left(k B-K_{X}\right)\right|_{H_{i}}$ is pseudoeffective for each $i$ and that $k B-V$ is pseudo-effective. ${ }^{3}$

We have the following numerical results.

Lemma 4.2. $K_{X} B^{n-1}>0$.

Proof. By the assumption, we know that $B^{n-1} \sim_{\text {num }} \operatorname{deg}_{Y}(M) \cdot C$ as one cycles on $X$, where $\operatorname{deg}_{Y}(M)=M^{n-1}>0$. Hence

$$
K_{X} B^{n-1}=\operatorname{deg}_{Y}(M)\left(K_{X} C\right)=(2 g-2) M^{n-1}>0 .
$$

The proof is finished.

Lemma 4.3. For any $0 \leq j \leq n-2$, we have

$$
K_{X} L^{n-1-j} B^{j} \leq 2 k K_{X} L^{n-2-j} B^{j+1}
$$

\footnotetext{
${ }^{3}$ Such an integer $k>0$ exists, since $\left.f\right|_{H_{i}}$ is generically finite and thus $\left.B\right|_{H_{i}}$ is nef and big for any $i$. Also, because $V$ is vertical, it has to be contained in the pullback of certain divisor on $Y$. Hence, increasing $k$, we may assume that $k B-V$ is pseudo-effective.
} 
Proof. Note that $K_{X}, L$, and $B$ are all nef, and $\left.\left(k B-K_{X}\right)\right|_{H_{i}}, k B-V$ are both pseudo-effective. It follows that

$$
\begin{aligned}
K_{X} L^{n-1-j} B^{j} & \leq K_{X}^{2} L^{n-2-j} B^{j} \\
& =\sum_{i=1}^{I_{0}} q_{i} K_{X} L^{n-2-j} B^{j} H_{i}+K_{X} L^{n-2-j} B^{j} V \\
& \leq \sum_{i=1}^{I_{0}} k q_{i} L^{n-2-j} B^{j+1} H_{i}+k K_{X} L^{n-2-j} B^{j+1} \\
& \leq 2 k K_{X} L^{n-2-j} B^{j+1} .
\end{aligned}
$$

This ends the proof.

Theorem 4.4. Under Assumption 4.1. Assume that $C$ is hyperelliptic. Let $\pi: X^{\prime} \rightarrow X$ be any birational morphism and $L^{\prime} \leq \pi^{*} L$ be any nef Cartier divisor on $X^{\prime}$.

- If $g \geq 3$, then

$$
h^{0}\left(L^{\prime}\right)-\frac{3\left(\pi^{*} K_{X}\right)\left(L^{\prime}\right)^{n-1}}{8(n-1) !} \leq n(2 k+2)^{n-2} K_{X} B^{n-1} .
$$

- If $g \geq 4$, then

$$
h^{0}\left(L^{\prime}\right)-\frac{\left(\pi^{*} K_{X}\right)\left(L^{\prime}\right)^{n-1}}{3(n-1) !} \leq n(2 k+2)^{n-2} K_{X} B^{n-1} .
$$

Proof. The proofs of the two inequalities are almost identical. We will give the detailed proof of the first one and leave the other to the reader.

We prove this theorem by induction. When $n=2$, i.e., $X$ is a fibered surface, we know that $\left(\pi^{*} K_{X}\right)\left(\pi^{*} C\right)=K_{X} C=2 g-2$. By Theorem 3.2, we have

$$
h^{0}\left(L^{\prime}\right)-\frac{3}{8}\left(\pi^{*} K_{X}\right) L^{\prime} \leq \frac{3}{8}(4 g-4) .
$$

Since $K_{X} B=(2 g-2) \operatorname{deg} M \geq 2 g-2$, we deduce that

$$
h^{0}\left(L^{\prime}\right)-\frac{3}{8}\left(\pi^{*} K_{X}\right) L^{\prime} \leq \frac{3}{4} K_{X} B<2 K_{X} B .
$$

This verifies the result for $n=2$. 
Now, suppose that the theorem holds when $\operatorname{dim} X \leq n-1(n \geq 3)$. We first show that

$$
h^{0}(L)-\frac{3 K_{X} L^{n-1}}{8(n-1) !} \leq \alpha_{n} K_{X} B^{n-1},
$$

where

$$
\alpha_{n}=n(2 k+2)^{n-2} .
$$

Choose a general pencil in $|B|$ and denote by $\sigma: X_{0} \rightarrow X$ the blow-up of the indeterminacies of this pencil. Then $X_{0}$ is a fibered variety over $\mathbb{P}^{1}$ whose general fiber $F$ is isomorphic to the general member of this chosen pencil.

Let $L_{0}=\sigma^{*} L, P_{0}=\sigma^{*}\left(K_{X}+B\right)$, and $B_{0}=\sigma^{*} B$. Applying Theorem 2.1 to $X_{0}, L_{0}$ and $P_{0}$, we obtain the following quadruples

$$
\left\{\left(X_{i}, L_{i}, Z_{i}, a_{i}\right): i=0, \ldots, N\right\}
$$

and $P_{i}$ 's which satisfy the conditions therein. Moreover, by Proposition 2.4, we have the following inequalities:

$$
\begin{aligned}
h^{0}(L) & =h^{0}\left(L_{0}\right) \leq \sum_{i=0}^{N} a_{i} r_{i}, \\
\left(K_{X}+B\right) L^{n-1} & =P_{0} L_{0}^{n-1} \geq(n-1) \sum_{i=0}^{N} a_{i} d_{i}-(n-1) d_{0} .
\end{aligned}
$$

Here

$$
r_{i}=h^{0}\left(\left.L_{i}\right|_{F_{i}}\right), \quad d_{i}=\left(\left.P_{i}\right|_{F_{i}}\right)\left(\left.L_{i}\right|_{F_{i}}\right)^{n-2} .
$$

In the following, we will compare $r_{i}$ and $d_{i}$ by induction.

First, we assume that $K_{X} L^{n-2} B>0$. This implies that

$$
d_{0}=\left(\left.P_{0}\right|_{F}\right)\left(\left.L_{0}\right|_{F}\right)^{n-2}=\left(K_{X}+B\right) L^{n-2} B \geq K_{X} L^{n-2} B>0 .
$$

Note that for each $i, F_{i}$ has a fibered structure by curves of genus $g$ induced by $f$. Moreover, by adjunction, $\left.P_{0}\right|_{F}=K_{F}$. Hence by induction on 
the dimension, we can get the following numerical inequality for each $i$ :

$$
r_{i}-\frac{3 d_{i}}{8(n-2) !} \leq \alpha_{n-1} K_{F}\left(\left.B\right|_{F}\right)^{n-2}=\alpha_{n-1} K_{X} B^{n-1} .
$$

Since $d_{0}>0$ and $L \leq K_{X}$, by Lemma 2.5 and Lemma 4.3, we obtain

$$
\sum_{i=0}^{N} a_{i}-1 \leq \frac{P_{0} L_{0}^{n-1}}{d_{0}} \leq \frac{K_{X} L^{n-1}+L^{n-1} B}{K_{X} L^{n-2} B} \leq \frac{K_{X} L^{n-1}}{K_{X} L^{n-2} B}+1 \leq 2 k+1,
$$

i.e.,

$$
\sum_{i=0}^{N} a_{i} \leq 2 k+2
$$

Applying Lemma 4.3 repeatedly, we get

$$
\begin{aligned}
L^{n-1} B & \leq K_{X} L^{n-2} B \leq(2 k)^{n-2} K_{X} B^{n-1}, \\
L^{n-2} B^{2} & \leq K_{X} L^{n-3} B^{2} \leq(2 k)^{n-3} K_{X} B^{n-1} .
\end{aligned}
$$

In particular, (4.7) and (4.8) imply that

$$
d_{0}=\left(\left.P_{0}\right|_{F}\right)\left(\left.L_{0}\right|_{F}\right)^{n-2}=\left(K_{X}+B\right) L^{n-2} B<2 \cdot(2 k)^{n-2} K_{X} B^{n-1} .
$$

Combine (4.3)-(4.9) together. It follows that

$$
\begin{aligned}
h^{0}(L)-\frac{3 K_{X} L^{n-1}}{8(n-1) !} \leq & \sum_{i=0}^{N}\left(r_{i}-\frac{3 d_{i}}{8(n-2) !}\right) a_{i}+\frac{3 d_{0}}{8(n-2) !}+\frac{3 L^{n-1} B}{8(n-1) !} \\
< & \alpha_{n-1} K_{X} B^{n-1} \sum_{i=0}^{N} a_{i} \\
& +\left(\frac{3(2 k)^{n-2}}{4(n-2) !}+\frac{3(2 k)^{n-2}}{8(n-1) !}\right) K_{X} B^{n-1} \\
\leq & (2 k+2) \alpha_{n-1} K_{X} B^{n-1}+\frac{(2 k)^{n-2}}{(n-2) !} K_{X} B^{n-1} \\
< & \alpha_{n} K_{X} B^{n-1} .
\end{aligned}
$$

In the last step, we use the fact that

$$
(2 k+2) \alpha_{n-1}+\frac{(2 k)^{n-2}}{(n-2) !} \leq(n-1)(2 k+2)^{n-2}+(2 k)^{n-2}<\alpha_{n} .
$$


Second, we assume that $K_{X} L^{n-2} B=0$. In this case, we have

$$
h^{0}(L-B)=0
$$

Otherwise, $L-B$ is effective. By Lemma 4.2, we would have

$$
K_{X} L^{n-2} B=K_{X} L^{n-3}(L-B+B) B \geq K_{X} L^{n-3} B^{2} \geq \cdots \geq K_{X} B^{n-1}>0,
$$

which is absurd. As a result, if we choose a general member $W \in|B|$, we get

$$
h^{0}(L) \leq h^{0}\left(\left.L\right|_{W}\right)
$$

Since $W$ itself is also fibered by curves of genus $g$ and $\left.L\right|_{W} \leq K_{W}$, by induction, we know that

$$
h^{0}(L) \leq h^{0}\left(\left.L\right|_{W}\right) \leq \frac{3 K_{W}\left(\left.L\right|_{W}\right)^{n-2}}{8(n-2) !}+\alpha_{n-1} K_{W}\left(\left.B\right|_{W}\right)^{n-2} .
$$

By adjunction, $K_{W}=\left.\left(K_{X}+B\right)\right|_{W}$. It follows that

$$
K_{W}\left(\left.B\right|_{W}\right)^{n-2}=\left(K_{X}+B\right) B^{n-1}=K_{X} B^{n-1}
$$

Also, using Lemma 4.3 repeatedly, we have

$$
\begin{aligned}
K_{W}\left(\left.L\right|_{W}\right)^{n-2} & =\left(K_{X}+B\right) L^{n-2} B=L^{n-2} B^{2} \\
& \leq K_{X} L^{n-3} B^{2} \leq(2 k)^{n-3} K_{X} B^{n-1}
\end{aligned}
$$

Combine (4.10)-(4.12) together, and we get

$$
h^{0}(L) \leq\left(\frac{3 \cdot(2 k)^{n-3}}{8(n-2) !}+\alpha_{n-1}\right) K_{X} B^{n-1} \leq \alpha_{n} K_{X} B^{n-1} .
$$

This finishes the proof of (4.2).

Now, let $\pi: X^{\prime} \rightarrow X$ be a birational morphism. Let $B^{\prime}=\pi^{*} B$. We can check that $k B^{\prime}-\pi^{*} V$ is still pseudo-effective. Moreover, $\left.\left(k B^{\prime}-\pi^{*} K_{X}\right)\right|_{\pi^{*} H_{i}}$ is also pseudo-effective for each $i$. Using the method in the proof of (4.2) verbatim, one can get

$$
h^{0}\left(L^{\prime}\right)-\frac{3\left(\pi^{*} K_{X}\right)\left(L^{\prime}\right)^{n-1}}{8(n-1) !} \leq \alpha_{n}\left(\pi^{*} K_{X}\right)\left(B^{\prime}\right)^{n-1}=\alpha_{n} K_{X} B^{n-1} .
$$

This finishes the whole proof. 
Theorem 4.5. Under Assumption 4.1. Assume that there is no family of hyperelliptic curves on $X$. Let $\pi: X^{\prime} \rightarrow X$ be a birational morphism. Then for any nef Cartier divisor $L^{\prime} \leq \pi^{*} L$, we have

$$
h^{0}\left(L^{\prime}\right)-\frac{\left(\pi^{*} K_{X}\right)\left(L^{\prime}\right)^{n-1}}{3(n-1) !} \leq \frac{7}{3} n(2 k+2)^{n-2} K_{X} B^{n-1} .
$$

Proof. The induction method in Theorem 4.4 applies here verbatim. If $n=2$, i.e., $X$ is a fibered surface, then by Proposition 3.1,

$$
h^{0}\left(L^{\prime}\right)-\frac{1}{3}\left(\pi^{*} K_{X}\right) L^{\prime} \leq \frac{7}{3} .
$$

This verifies the theorem when $n=2$.

With the above result, by the same induction method as in Theorem 4.4, we can get the conclusion. We leave the detailed proof to the reader.

The direct application of the above theorems is when $X^{\prime}=X$ and $L^{\prime}=$ $K_{X}$. We have the following summarized result.

Theorem 4.6. Suppose that we are under Assumption 4.1.

- If $C$ is hyperelliptic of genus $g \geq 3$, then

$$
h^{0}\left(K_{X}\right)-\frac{3 K_{X}^{n}}{8(n-1) !} \leq n(2 k+2)^{n-2} K_{X} B^{n-1} .
$$

- If $C$ is hyperelliptic of genus $g \geq 4$, then

$$
h^{0}\left(K_{X}\right)-\frac{K_{X}^{n}}{3(n-1) !} \leq n(2 k+2)^{n-2} K_{X} B^{n-1} .
$$

- If there is no family of hyperelliptic curves on $X$, then

$$
h^{0}\left(K_{X}\right)-\frac{K_{X}^{n}}{3(n-1) !} \leq \frac{7}{3} n(2 k+2)^{n-2} K_{X} B^{n-1} .
$$

Proof. We just prove the first inequality. The others are almost the same. 
Let $\pi: X^{\prime} \rightarrow X$ be a birational modification such that the movable part of $\pi^{*} K_{X}$ is base point free, i.e., we can write

$$
\pi^{*} K_{X}=L^{\prime}+Z^{\prime}
$$

where $\left|L^{\prime}\right|$ is base point free, $Z^{\prime} \geq 0$ is a $\mathbb{Q}$-divisor, and $h^{0}\left(L^{\prime}\right)=h^{0}\left(K_{X}\right)$. Apply Theorem 4.4 to $X^{\prime}$ and $L^{\prime}$. It follows that

$$
h^{0}\left(K_{X}\right)-\frac{3\left(\pi^{*} K_{X}\right)\left(L^{\prime}\right)^{n-1}}{8(n-1) !} \leq n(2 k+2)^{n-2} K_{X} B^{n-1} .
$$

Note that

$$
\left(\pi^{*} K_{X}\right)\left(L^{\prime}\right)^{n-1} \leq\left(\pi^{*} K_{X}\right)^{n}=K_{X}^{n}
$$

We can easily get the desired result.

\section{Proof of the main theorem}

In this section, we prove the main theorem.

Theorem 5.1. Let $X$ be an $n$-dimensional normal, irregular minimal $\mathbb{Q}$ Gorenstein variety of general type. Assume $X$ has one dimensional Albanese fiber.

(1) The general Albanese fiber is of genus 2 provided that

$$
K_{X}^{n}<\frac{8}{3}(n-1) ! \chi\left(\omega_{X}\right)
$$

(2) The general Albanese fiber is hyperelliptic of genus 2 or 3 provided that

$$
K_{X}^{n}<3(n-1) ! \chi\left(\omega_{X}\right)
$$

Proof. Our proof is based on the following three ingredients: (1) Theorem 4.6, (2) the covering and limiting method by Pardini [9], and (3) the generic vanishing theorem by Green and Lazarsfeld [6]. We give the proof by steps.

Step 1: Construction of covers and set up. Let $A$ be the Albanese variety of $X$, and $\alpha=\operatorname{Alb}_{X}: X \rightarrow A$ be the Albanese map of $X$. For any $d \in \mathbb{Z}_{>0}$, denote by $\mu_{d}$ the multiplication map of $A$ by $d$. Let $X_{d}=X \times_{\mu_{d}} A$. 
Then we have the following commutative diagram:

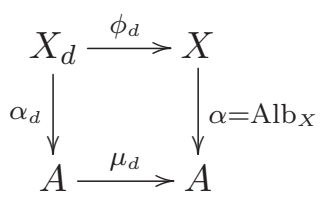

Note that $X_{d}$ is always irreducible, because the induced map $\alpha^{*}: \operatorname{Pic}^{0}(A) \rightarrow$ $\operatorname{Pic}^{0}(X)$ is injective, and in fact, an isomorphism here.

Fix a sufficiently ample Cartier divisor $H$ on $A$. Let $B=\alpha^{*} H$ and $B_{d}=$ $\alpha_{d}^{*} H$ for any $d \geq 1$. By [3, Chapter 2, Proposition 3.5], we have the following numerical equivalence:

$$
d^{2} B_{d} \sim_{\text {num }} \phi_{d}^{*} B
$$

This implies that for any $1 \leq i \leq n$,

$$
K_{X_{d}}^{n-i} B_{d}^{i}=\left(\phi_{d}^{*} K_{X}\right)^{n-i}\left(\frac{1}{d^{2}} \phi_{d}^{*} B\right)^{i}=d^{2 m-2 i} K_{X}^{n-i} B^{i} .
$$

Here $m=h^{1}\left(\mathcal{O}_{X}\right)=\operatorname{dim} A$.

For each $d>0$, let $X_{d} \stackrel{f_{d}}{\longrightarrow} Y_{d} \stackrel{h_{d}}{\longrightarrow} A$ be the Stein factorization of $\alpha_{d}$. Let $M_{d}=h_{d}^{*} H$. Thus $B_{d}=f_{d}^{*} M_{d}$.

Step 2: Reduction to the case of positive genus. Note that to prove the theorem, we can assume that $\chi\left(\omega_{X}\right)>0$. Since $\operatorname{dim} A=m$ and $X$ has one dimensional Albanese fiber, we have $m \geq n-1$.

Notice that $\phi_{d}$ is étale. Thus it follows that

$$
d^{2 m} \chi\left(\omega_{X}\right)=\chi\left(\omega_{X_{d}}\right)=\sum_{i=0}^{n}(-1)^{i} h^{i}\left(\omega_{X_{d}}\right) \leq h^{0}\left(K_{X_{d}}\right)+\sum_{i=2}^{n}(-1)^{i} h^{i}\left(\omega_{X_{d}}\right) .
$$

On the other hand, by [14, Theorem 4.1], which is in the spirit of the generic vanishing theorem of Green and Lazarsfeld [6], for each $i=0, \ldots, n-2$, we have

$$
\lim _{d \rightarrow \infty} \frac{h^{i}\left(\mathcal{O}_{X_{d}}\right)}{d^{2 m}}=0 .
$$

By duality, it implies that $h^{i}\left(\omega_{X_{d}}\right) \sim o\left(d^{2 m}\right)$ for $i \geq 2$. Hence

$$
h^{0}\left(K_{X_{d}}\right) \geq d^{2 m} \chi\left(\omega_{X}\right)+o\left(d^{2 m}\right) .
$$

In particular, we can assume that $h^{0}\left(K_{X_{d}}\right)>0$ when $d$ is large. Since it suffices to prove this theorem on any étale cover, we can assume that $h^{0}\left(K_{X}\right)>0$. 
Step 3: Pseudo-effectiveness. As in (4.1), we can write

$$
K_{X} \sim_{\operatorname{lin}_{\mathbb{Q}}} \sum_{i=1}^{I_{0}} q_{i} H_{i}+V
$$

where $q_{i} \in \mathbb{Q}_{>0}$, each $H_{i} \geq 0$ is an irreducible, reduced and horizontal divisor, and $V \geq 0$ is the vertical part. We can also find an integer $k>0$ such that $\left.\left(k B-K_{X}\right)\right|_{H_{i}}$ is pseudo-effective for each $i$ and that $k B-V$ is pseudo-effective.

Since $\phi_{d}$ is étale, we know that $K_{X_{d}}=\phi_{d}^{*} K_{X}$. Hence we can write

$$
K_{X_{d}} \sim_{\operatorname{lin} \mathbb{Q}} \sum_{i=1}^{I_{0}} q_{i} \phi_{d}^{*} H_{i}+\phi_{d}^{*} V=\sum_{i=1}^{I_{0}} q_{i}\left(\sum_{j=1}^{l_{d}} H_{i, j}\right)+\phi_{d}^{*} V
$$

Here each $H_{i, j}$ is an irreducible and reduced component of the divisor $\phi_{d}^{*} H_{i}$ on $X_{d}$.

By pulling back, we know that

$$
\left.\left(k \phi_{d}^{*} B-K_{X_{d}}\right)\right|_{H_{i, j}} \quad \text { and } \quad k \phi_{d}^{*} B-\phi_{d}^{*} V
$$

are pseudo-effective. Because $d^{2} B_{d} \sim_{\text {num }} \phi_{d}^{*} B$, we deduce that

$$
\left.\left(k d^{2} B_{d}-K_{X_{d}}\right)\right|_{H_{i, j}} \quad \text { and } \quad k d^{2} B_{d}-\phi_{d}^{*} V
$$

are pseudo-effective.

Step 4: Hyperelliptic case. If the general Albanese fiber of $X$ is hyperelliptic of genus $g \geq 3$, so is the general fiber of $f_{d}$. By the first inequality in Theorem 4.6, we can get

$$
h^{0}\left(K_{X_{d}}\right)-\frac{3 K_{X_{d}}^{n}}{8(n-1) !} \leq n\left(2 k d^{2}+2\right)^{n-2} K_{X_{d}} B_{d}^{n-1}
$$

Recall that by (5.1), we have

$$
K_{X_{d}}^{n}=d^{2 m} K_{X}^{n}, \quad K_{X_{d}} B_{d}^{n-1}=d^{2 m-2 n+2} K_{X} B^{n-1} .
$$

Combine this with (5.2), and it follows that

$$
d^{2 m} \chi\left(\omega_{X}\right)+o\left(d^{2 m}\right)-\frac{3 d^{2 m} K_{X}^{n}}{8(n-1) !} \leq n\left(2 k d^{2}+2\right)^{n-2} d^{2 m-2 n+2} K_{X} B^{n-1} .
$$


Letting $d \rightarrow \infty$, we deduce that

$$
K_{X}^{n} \geq \frac{8}{3}(n-1) ! \chi\left(\omega_{X}\right)
$$

By the same method and applying the second inequality in Theorem 4.6, we deduce that if the Albanese fiber is hyperelliptic of genus $g \geq 4$, then

$$
K_{X}^{n} \geq 3(n-1) ! \chi\left(\omega_{X}\right)
$$

Step 5: Non-hyperelliptic case. Now assume that the general Albanese fiber of $X$ is non-hyperelliptic. So is the general fiber of $f_{d}$.

Step 5.1. We first show that, up to étale covers, we have

$$
h^{0}\left(K_{X}-B\right)>0 .
$$

If this is true, then $\alpha^{*}|H|$ can be identified as a subspace of $\left|K_{X}\right|$. Note that $H$ is sufficiently ample. Thus the map given by this (non-complete) linear system $\alpha^{*}|H|$ is identical to the Albanese map of $X$. It follows that the Albanese map of $X$ factors through the canonical map of $X$.

Now let us prove (5.3). Choose a very general member $W \in\left|B_{d}\right|$. Since $h^{0}\left(K_{X_{d}}\right)>0$, by adjunction, $h^{0}\left(K_{W}\right)>0$. Hence we can consider the canonical map of $W$. We have the following diagram:

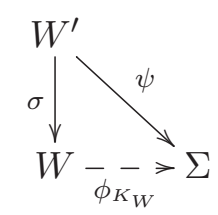

where $\Sigma \subseteq \mathbb{P}^{h^{0}\left(K_{W}\right)-1}$ is the canonical image of $W$, and $\sigma$ is the blow-up of the indeterminacies of $\left|K_{W}\right|$. By Lemma 5.2, we have

$$
h^{0}\left(\left.K_{X_{d}}\right|_{W}\right) \leq h^{0}\left(K_{W}\right) \leq\left(\mathcal{O}_{\Sigma}(1)\right)^{\operatorname{dim} \Sigma}+\operatorname{dim} \Sigma .
$$

On the other hand, let $l \in \mathbb{Z}_{>0}$ be a positive integer such that $l K_{X}$ is Cartier. By adjunction, $l K_{W}$ is also Cartier. Note that $K_{W}$ is nef and big. We have

$$
\begin{aligned}
K_{W}^{n-1} & =\left(\sigma^{*} K_{W}\right)^{n-1} \geq\left(\sigma^{*} K_{W}\right)^{n-1-\operatorname{dim} \Sigma}\left(\psi^{*} \mathcal{O}_{\Sigma}(1)\right)^{\operatorname{dim} \Sigma} \\
& \geq \frac{1}{l^{n-1-\operatorname{dim} \Sigma}}\left(\mathcal{O}_{\Sigma}(1)\right)^{\operatorname{dim} \Sigma} .
\end{aligned}
$$


Hence it follows from (5.1), (5.4) and (5.5) that

$$
\begin{aligned}
h^{0}\left(K_{X_{d}} \mid W\right) & \leq l^{n-1-\operatorname{dim} \Sigma} K_{W}^{n-1}+\operatorname{dim} \Sigma \\
& \leq l^{n-1-\operatorname{dim} \Sigma}\left(K_{X_{d}}+B_{d}\right)^{n-1} B_{d}+n-1 \sim o\left(d^{2 m}\right) .
\end{aligned}
$$

Therefore, up to étale covers, we can assume that

$$
h^{0}\left(K_{X}\right)-h^{0}\left(\left.K_{X}\right|_{W}\right)>0
$$

for a general member $W \in|B|$. By the following exact sequence

$$
0 \longrightarrow H^{0}\left(\mathcal{O}_{X}\left(K_{X}-B\right)\right) \longrightarrow H^{0}\left(\mathcal{O}_{X}\left(K_{X}\right)\right) \longrightarrow h^{0}\left(\mathcal{O}_{W}\left(K_{X}\right)\right)
$$

we know that

$$
h^{0}\left(K_{X}-B\right)>0 .
$$

Note that in the meantime, we also obtain

$$
h^{0}\left(K_{X_{d}}-B_{d}\right)>0
$$

for any $d>0$, which implies that the map $\alpha_{d}$ factors through the canonical map of $X_{d}$. That is, we have the following commutative diagram:

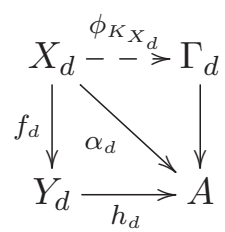

where $\Gamma_{d}$ is the canonical image of $X_{d}$.

Step 5.2. We claim that there is no family of hyperelliptic curves on $X_{d}$.

If the claim is not true, then there exists a hyperelliptic family on $X_{d}$. Furthermore, we have the following two possibilities: either this family is contracted by $\phi_{K_{X_{d}}}$ or not. If it is contracted by $\phi_{K_{X_{d}}}$, it would also be contracted by $\alpha_{d}$, thus by $f_{d}$. This is impossible since the general fiber of $f_{d}$ is non-hyperelliptic. The second case is still impossible. Because if this family is not contracted by $\phi_{K_{X_{d}}}$, then its image under $\phi_{K_{X_{d}}}$ is a family of $\mathbb{P}^{1}$ curves on $\Gamma_{d}$. Since $\alpha_{d}\left(X_{d}\right)$ can not have any $\mathbb{P}^{1}$ family, this hyperelliptic family has to be contracted by $\alpha_{d}$, thus by $f_{d}$. It contradicts our assumption again. 
Step 5.3. Since $X_{d}$ has no family of hyperelliptic curves, by the third inequality in Theorem 4.6, we have

$$
h^{0}\left(K_{X_{d}}\right)-\frac{K_{X_{d}}^{n}}{3(n-1) !} \leq \frac{7}{3} n\left(2 k d^{2}+2\right)^{n-2} K_{X_{d}} B_{d}^{n-1} .
$$

Use the method in Step 4 and let $d \rightarrow \infty$. It follows that

$$
K_{X}^{n} \geq 3(n-1) ! \chi\left(\omega_{X}\right)
$$

The proof will be completed by showing the following lemma which is undoubtedly known to experts.

Lemma 5.2. Let $L$ be a Cartier divisor on a projective variety $X$ such that $|L|$ is base point free. Let $\phi_{L}: X \rightarrow \mathbb{P}^{h^{0}(L)-1}$ be the morphism induced by $|L|$. Denote $\Sigma=\phi_{L}(X)$ and $d=\operatorname{dim} \Sigma$. Then

$$
h^{0}(L) \leq\left(\mathcal{O}_{\Sigma}(1)\right)^{d}+d
$$

Proof. Denote by $W$ a very general hyperplane section of $\Sigma$. We have

$$
0 \longrightarrow H^{0}\left(\mathcal{O}_{\Sigma}\right) \longrightarrow H^{0}\left(\mathcal{O}_{\Sigma}(1)\right) \longrightarrow H^{0}\left(\mathcal{O}_{W}(1)\right)
$$

It gives

$$
h^{0}(L)=h^{0}\left(\mathcal{O}_{\Sigma}(1)\right) \leq h^{0}\left(\mathcal{O}_{W}(1)\right)+1 .
$$

Repeat this $d-1$ times to get

$$
h^{0}(L) \leq h^{0}\left(\mathcal{O}_{C}(1)\right)+(d-1)
$$

where $C$ is the complete intersection of $d-1$ general hyperplane sections. In particular,

$$
\operatorname{deg} \mathcal{O}_{C}(1)=\left(\mathcal{O}_{\Sigma}(1)\right)^{d}
$$

Therefore,

$$
h^{0}\left(\mathcal{O}_{C}(1)\right) \leq\left(\mathcal{O}_{\Sigma}(1)\right)^{d}+1
$$

This ends the proof.

\section{Final Remark}

In [1, Remark 4.5], by using Xiao's method on the Harder-Narasimhan filtration, Barja shows the following: 
Theorem (Barja). Let $X$ be an $n$-dimensional normal, irregular minimal Gorenstein variety of general type with Albanese fiber dimension one. Then

$$
K_{X}^{n} \geq 2(n-1) ! \chi\left(\omega_{X}\right)
$$

In fact, using the proof of Theorem 1.1, we can give an alternative proof of this result. Moreover, our proof is also valid for $\mathbb{Q}$-Gorenstein varieties. The induction method can be used here verbatim. We only need to show the corresponding result for surfaces, which is nothing but the following Noether type result:

Proposition 6.1. Let $S^{\prime}$ be a minimal surface of general type, and $\sigma: S \rightarrow$ $S^{\prime}$ be a birational map. Then for any nef divisor $L \leq \sigma^{*} K_{S^{\prime}}$ on $S$, we have

$$
\left(\sigma^{*} K_{S^{\prime}}\right) L \geq 2 h^{0}(L)-4
$$

Proof. See [8, Lemmas 2.2, 2.3].

We leave the proof to the reader.

\section{Acknowledgements}

The author would like to thank the referee sincerely for reading the paper so carefully and particularly for many valuable suggestions that have improved the writing of this paper dramatically.

The author is supported by an NSERC Discovery Grant.

\section{References}

[1] M. A. Barja, Generalized Clifford-Severi inequality and the volume of irregular varieties, Duke Math. J. 164 (2015), no. 3, 541-568.

[2] A. Beauville, L'application canonique pour les surfaces de type général, Invent. Math. 55 (1979), no. 2, 121-140.

[3] C. Birkenhake and H. Lange, Complex abelian varieties, Vol. 302 of Grundlehren der Mathematischen Wissenschaften [Fundamental Principles of Mathematical Sciences], Springer-Verlag, Berlin, second edition (2004), ISBN 3-540-20488-1.

[4] E. Bombieri, Canonical models of surfaces of general type, Inst. Hautes Études Sci. Publ. Math. (1973), no. 42, 171-219. 
[5] O. Debarre, Inégalités numériques pour les surfaces de type général, Bull. Soc. Math. France 110 (1982), no. 3, 319-346. With an appendix by A. Beauville.

[6] M. Green and R. Lazarsfeld, Deformation theory, generic vanishing theorems, and some conjectures of Enriques, Catanese and Beauville, Invent. Math. 90 (1987), no. 2, 389-407.

[7] E. Horikawa, Algebraic surfaces of general type with small $c_{1}^{2}$. V, J. Fac. Sci. Univ. Tokyo Sect. IA Math. 28 (1981), no. 3, 745-755 (1982).

[8] K. Ohno, Some inequalities for minimal fibrations of surfaces of general type over curves, J. Math. Soc. Japan 44 (1992), no. 4, 643-666.

[9] R. Pardini, The Severi inequality $K^{2} \geq 4 \chi$ for surfaces of maximal Albanese dimension, Invent. Math. 159 (2005), no. 3, 669-672.

[10] M. Reid, Quadrics through a canonical surface, in Algebraic geometry (L'Aquila, 1988), Vol. 1417 of Lecture Notes in Math., 191-213, Springer, Berlin (1990).

[11] G. Xiao, Fibered algebraic surfaces with low slope, Math. Ann. 276 (1987), no. 3, 449-466.

[12] G. Xiao, Hyperelliptic surfaces of general type with $K^{2}<4 \chi$, Manuscripta Math. 57 (1987), no. 2, 125-148.

[13] X. Yuan and T. Zhang, Relative Noether inequality on fibered surfaces, Adv. Math. 259 (2014) 89-115.

[14] T. Zhang, Severi inequality for varieties of maximal Albanese dimension, Math. Ann. 359 (2014), no. 3-4, 1097-1114.

[15] T. Zhang, Geography of Irregular Gorenstein 3-folds, Canad. J. Math. 67 (2015), no. 3, 696-720.

Department of Mathematics, University of Alberta

Edmonton, Alberta T6G 2G1, Canada

Current address:

Department of Mathematical Sciences, Durham University

Durham, DH1 3LE, United Kingdom

E-mail address: tong.zhang@durham.ac.uk, mathtzhang@gmail.com

Received September 17, 2014 